\title{
Genetically Encoded Green Fluorescent Biosensors for Monitoring UDP-GIcNAc in Live Cells
}

\author{
Zefan Li, Jing Zhang, and Hui-wang Ai*
}

Cite This: ACS Cent. Sci. 2021, 7, 1763-1770

Read Online

ABSTRACT: Uridine diphosphate $N$-acetylglucosamine (UDPGlcNAc) is a nucleotide sugar used by glycosyltransferases to synthesize glycoproteins, glycosaminoglycans, glycolipids, and glycoRNA. UDP-GlcNAc also serves as the donor substrate for forming $\mathrm{O}$-GlcNAc, a dynamic intracellular protein modification involved in diverse signaling and disease processes. UDP-GlcNAc is thus a central metabolite connecting nutrition, metabolism, signaling, and disease. There is a great interest in monitoring UDPGlcNAc in biological systems. Here, we present the first genetically

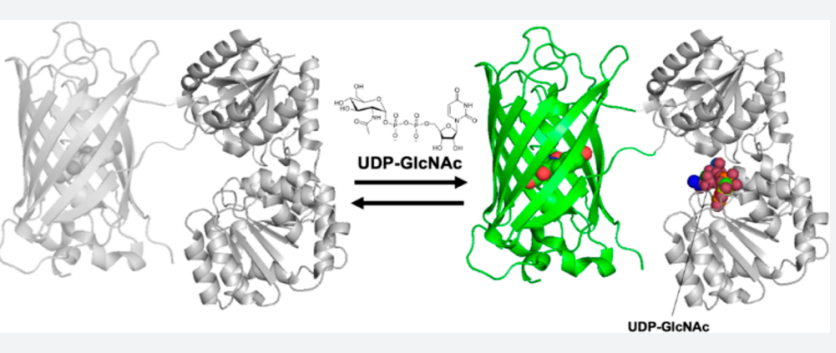
encoded, green fluorescent UDP-GlcNAc sensor (UGAcS), an optimized insertion of a circularly permuted green fluorescent protein (cpGFP) into an inactive mutant of an Escherichia coli UDP-GlcNAc transferase, for ratiometric monitoring of UDP-GlcNAc dynamics in live mammalian cells. Although UGAcS responds to UDP-GlcNAc quite selectively among various nucleotide sugars, UDP and uridine triphosphate (UTP) interfere with the response. We thus developed another biosensor named UXPS, which is responsive to UDP and UTP but not UDP-GlcNAc. We demonstrated the use of the biosensors to follow UDP-GlcNAc levels in cultured mammalian cells perturbed with nutritional changes, pharmacological inhibition, and knockdown or overexpression of key enzymes in the UDP-GlcNAc synthesis pathway. We further utilized the biosensors to monitor UDP-GlcNAc concentrations in pancreatic MIN6 $\beta$-cells under various culture conditions.

\section{INTRODUCTION}

Uridine diphosphate $N$-acetylglucosamine (UDP-GlcNAc), the major end-product of the hexosamine biosynthetic pathway (HBP), is one of the most important nucleotide sugars in living organisms. ${ }^{1}$ The HBP branches out from glycolysis and consumes $\sim 0.006-3 \%$ of total glucose,$^{2-4}$ along with glutamine, acetyl-coenzyme A (Ac-CoA), adenosine triphosphate (ATP), and uridine triphosphate (UTP). Because multiple types of metabolic molecules, including carbohydrates, amino acids, fatty acids, and nucleotides, regulate the flux of HBP, UDP-GlcNAc has been considered an integrator of nutritional and metabolic signals., ${ }^{5,6}$

As an activated $\mathrm{N}$-acetylglucosamine (GlcNAc) donor, UDP-GlcNAc is vital for the glycosyltransferase-catalyzed formation of various glycosaminoglycans, glycoproteins, glycolipids, and glycoRNAs. ${ }^{1,7}$ In mammalian cells, glycosylation primarily occurs in the endoplasmic reticulum (ER) and Golgi. The glycosylated products are typically translocated to the extracellular space, playing critical roles such as maintaining structural stability, modulating cell-matrix or cell-cell interaction, regulating cell proliferation and migration, and initiating other types of signaling. ${ }^{1,6}$ Moreover, UDPGlcNAc is an essential substrate for O-GlcNAcylation, a reversible post-translational modification of nucleocytoplasmic proteins. ${ }^{2,8,9}$ O-GlcNAc transferase (OGT) catalyzes the transfer of the GlcNAc subunit from UDP-GlcNAc to the serine or threonine residues of proteins, while O-GlcNAcase
(OGA) hydrolyzes the modification to generate free proteins and GlcNAc. This dynamic and tightly regulated process, analogous to more well-known phosphorylation, is involved in a large array of intracellular signaling processes. ${ }^{1,5,6,10-12}$ Aberrant $\mathrm{O}$-GlcNAcylation has been linked to aging, neurodegeneration, cancer, cardiovascular diseases, and metabolic disorders. ${ }^{2,6,13-15}$

The concentration of UDP-GlcNAc is one of the several key factors regulating glycosylation. The drastic increase of the $\beta 1,6$-branched oligosaccharide levels was observed in B16 melanoma cells incubated with GlcNAc. ${ }^{16}$ In another example, deleterious mutations in SLC35A3, the major Golgi UDPGlcNAc transporter, were identified in patients with autism spectrum disorder, arthrogryposis, and epilepsy. ${ }^{17}$ These mutations reduce UDP-GlcNAc transport into the ER, leading to a massive decrease of highly branched $N$-glycans and a drastic increase of lower branched glycoforms at the cell surface. ${ }^{17}$ The UDP-GlcNAc level has also been found to regulate intracellular $O$-GlcNAcylation. In vitro character-

Received: June 22, 2021

Published: September 30, 2021 
A

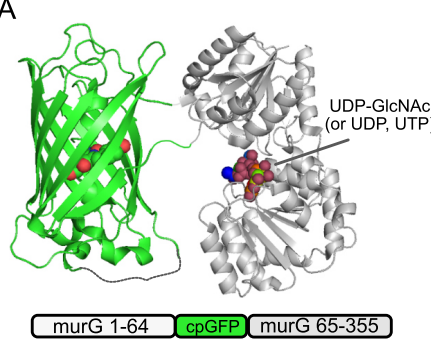

D

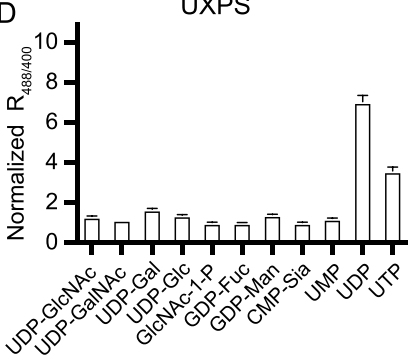

B
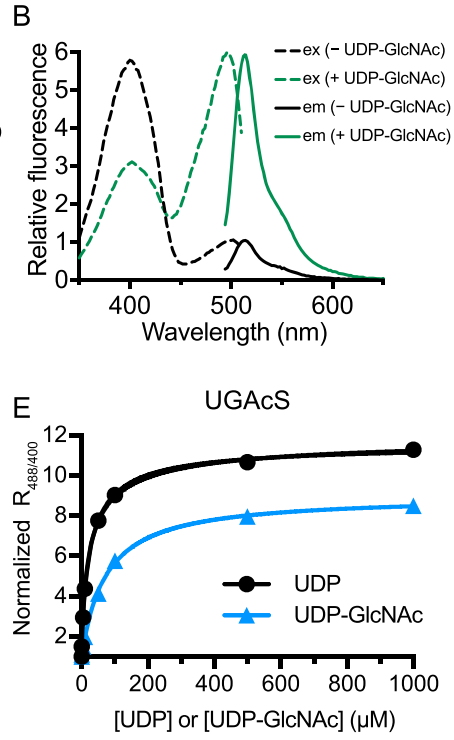

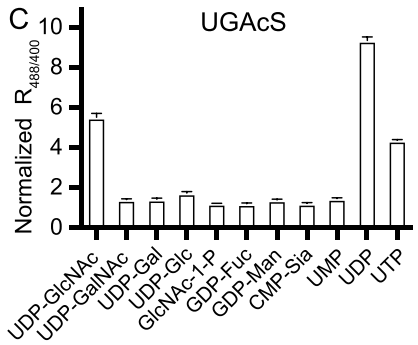

$\mathrm{F}$

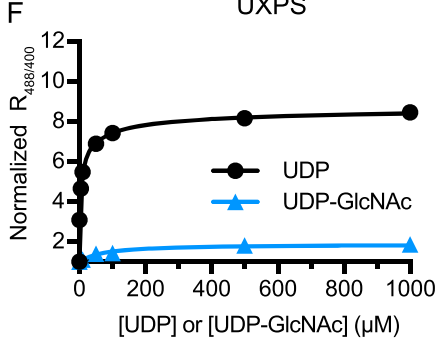

Figure 1. Design and in vitro characterization of the biosensors. (A) Illustration of the sensor design, showing the cpGFP insertion between residues 64 and 65 of the murG glycosyltransferase. Also highlighted is a UDP-GlcNAc molecule in the substrate-binding pocket. (B) Fluorescence excitation and emission spectra of UGAcS before and after addition of $1 \mathrm{mM}$ UDP-GlcNAc. Responses of UGAcS (C) or UXPS (D), presented as normalized fluorescence excitation ratios $(488 \mathrm{~nm} / 400 \mathrm{~nm})$, to $100 \mu \mathrm{M}$ of various nucleoside sugars and other related cellular metabolites. Data are presented as mean $\pm \mathrm{SD}$ ( $n=3$ technical repeats). Dose-dependent responses of UGAcS (E) or UXPS (F) to UDP or UDP-GlcNAc.

ization of OGT demonstrated that the concentration of UDPGlcNAc was positively correlated with the $O$-GlcNAcylation of the tested peptide substrates. ${ }^{18}$ Supplementing human hepatocellular carcinoma HepG2 cells with glucosamine, a metabolite used by HBP to synthesize UDP-GlcNAc, significantly increased O-GlcNAc. ${ }^{19,20}$ Furthermore, hyperglycemia was shown to increase the O-GlcNAc level in multiple tissue types via the HBP. ${ }^{21-23}$

Because of the importance of UDP-GlcNAc in metabolic sensing, signaling, and disease, methods for monitoring UDPGlcNAc levels in living systems are highly needed. Traditionally, chromatography methods are used to determine cellular UDP-GlcNAc levels, ${ }^{3,24,25}$ but these methods require cell lysis and provide little spatiotemporal resolution. To address this technical gap, we engineered the first genetically encoded fluorescent sensor, UGAcS, for detecting UDP-GlcNAc in living cells. We inserted a cpGFP into an inactive mutant of murG, ${ }^{26-28}$ an Escherichia coli UDP-GlcNAc transferase, and performed directed evolution to optimize the biosensor. Because UDP and UTP interfere with the response of UGAcS to UDP-GlcNAc, we developed an additional control biosensor, UXPS, which is only responsive to UDP and UTP. We demonstrated the use of the biosensors to follow UDPGlcNAc concentration changes in cultured mammalian cells in response to various nutritional, pharmacological, and genetic perturbations.

\section{RESULTS AND DISCUSSION}

Design, Engineering, and in Vitro Characterization of the UGACS. MurG is a well-characterized E. coli UDP-GlcNAc transferase involved in synthesizing lipid-linked precursors to assemble peptidoglycan, the polymeric cell wall outside the bacterial cell membrane. ${ }^{28,29}$ MurG has a high binding affinity to UDP-GlcNAc $(\sim 1.4 \mu \mathrm{M})$, and its structures in the apo and UDP-GlcNAc-bound forms have been reported. ${ }^{27,29} \mathrm{We}$ selected murG as the sensory domain to build a UDP-GlcNAc sensor. By carefully examining the structures of murG, we identified that the binding of UDP-GlcNAc triggers a structural conversion of residues 60-70 in murG from a loop into an $\alpha$ helix (Supporting Information, Figure S1). We further confirmed the significant conformation change at this loop by analyzing the changes of dihedral angles of every four consecutive $\mathrm{C}_{\alpha}$ atoms (Figure S2).

We next inserted cpGFP to the above-identified loop between residues 64 and 65 of murG (Figure $1 \mathrm{~A}$ and Figure S3). A fully randomized residue was introduced to each of the two junctions as the linkers. We screened the library and identified a UGAcS0.1 mutant with a $30 \%$ response $((R-$ $\left.R_{0}\right) / R_{0}$ or $\Delta R / R_{0}$, where $R$ is the ratio of fluorescence with 488 $\mathrm{nm}$ excitation to that with $400 \mathrm{~nm}$ excitation) to UDPGlcNAc. To increase the sensor's response to UDP-GlcNAc, we performed three rounds of error-prone PCR, and the screening of these libraries resulted in UGAcS0.2 showing a $300 \%\left(\Delta R / R_{0}\right)$ response.

Although our primary goal is to apply the fluorescent biosensors in mammalian cells, and murG is unlikely to be active in mammalian cells where its lipid-linked peptidoglycan substrate is not present, we still performed saturation mutagenesis on the catalytic His19 residue of murG in UGAcS0.2. This residue is essential for catalysis and conserved in murG from 73 orthologues. ${ }^{26,27}$ Our screening of the mutants led to an enzymatically inactive His19Ser mutant (UGAcS0.3) but is comparable to UGAcS0.2 in terms of the UDP-GlcNAc responsiveness.

Because UDP is a natural inhibitor and regulator of UDPsugar transferases, we examined the fluorescence of UGAcS0.3 upon the addition of UDP. UGAcS0.3 showed a higher response to UDP than UDP-GlcNAc. Therefore, we next devoted our effort to engineering UGAcS0.3 for increased specificity to UDP-GlcNAc versus UDP. We chose five pairs of residues (residues 192 and 193, residues 16 and 127, residues 164 and 269, or residues 244 and 245) in the ligand-binding 
pocket and performed saturation mutagenesis. Screening of these libraries resulted in UGAcS0.4 with mutations at residues 192 and 193. In comparison with UGAcS0.3, UGAcS0.4 showed increased responsiveness to UDP-GlcNAc and reduced responsiveness to UDP. Despite the progress, we were unable to identify a mutant to exclude the UDP interference entirely.

From UGAcS0.4, we performed two more rounds of random mutagenesis. By screening these libraries for improved UDPGlcNAc responsiveness, we arrived at UGAcS, which showed a nearly $700 \%$ response $\left(\Delta R / R_{0}\right)$ to $1 \mathrm{mM}$ UDP-GlcNAc (Figure 1B and Figure S4). We further tested the specificity of UGAcS using various nucleotide sugars and other related compounds at physiologically relevant concentrations (Figure 1C). UGAcS responded to UDP-GlcNAc, UDP, and UTP. Other tested nucleotide sugars, including uridine diphosphate $\mathrm{N}$-acetylgalactosamine (UDP-GalNAc) and uridine diphosphate glucose (UDP-Glc) that are structurally very close to UDP-GlcNAc, induced little fluorescence change.

After we realized that it might be unrealistic to engineer a fully specific UDP-GlcNAc biosensor from the glycosyltransferase, we sought to identify a UDP sensor, which could be used as a control to cross-check the responses of the UGAcS variants. From one of the ligand-binding-pocket mutagenesis libraries mentioned above, we identified a mutant (UXPS), which is responsive to UDP and UTP, not to UDP-GlcNAc and other nucleotide sugars (Figure 1D and Figure S4).

We next titrated UGAcS and UXPS with various concentrations of UDP-GlcNAc and UDP (Figure 1E,F). The apparent dissociation constant $\left(K_{\mathrm{d}}\right)$ values (deduced from the fluorescence responses of the sensors) for UGAcS were determined to be $72 \pm 4$ and $26 \pm 1 \mu \mathrm{M}$ in the presence of UDP-GlcNAc and UDP, respectively, while UXPS responded to UDP with an apparent $K_{\mathrm{d}}$ of $6 \pm 1 \mu \mathrm{M}$.

Monitoring of UDP-GICNAC Changes in HEK 293T Cells. 2-Deoxy-D-glucose (2-DG) is a glucose analogue, and 2deoxy-D-glucose-6-phosphate (2-DG6P) intracellularly formed from 2-DG competitively inhibits hexokinase (HK) and phosphoglucose isomerase (PGI), the enzymes involved in the first two steps of glycolysis (Figure $2 \mathrm{~A}$ ). ${ }^{30}$ To validate the function of UGAcS in mammalian cells, we expressed the sensors in human embryonic kidney (HEK) 293T cells and examined sensor responses to glycolysis inhibition by 2-DG. HEK293T cells transiently expressing UGAcS emitted strong green fluorescence. Upon $3 \mathrm{~h}$ of incubation with $10 \mathrm{mM} 2-\mathrm{DG}$, the ratio of the fluorescence with $488 \mathrm{~nm}$ excitation to that with $400 \mathrm{~nm}$ excitation (denoted as $R_{488 / 400}$ ) decreased by $27.6 \%$ (Figure 2B). The fluorescence change could be reversed by washing out 2-DG, confirming the reversibility of UGAcS. Meanwhile, we expressed the control sensor UXPS in HEK 293T cells and treated the cells using the same procedure. The fluorescence change of UXPS was minimal, and the magnitude of the difference was not statistically significant (Figure 2C).

To cross-verify the results, we adapted a hydrophilic interaction chromatography-mass spectrometry (HILIC-MS) method to quantify UDP-GlcNAc from cell extracts. ${ }^{3,31}$ Briefly, we prepared the lysates of HEK 293T cells treated or untreated with 2 -DG and doped in ${ }^{13} \mathrm{C}$-double-labeled UDP-GlcNAc $\left({ }^{13} \mathrm{C}_{2}\right.$-UDP-GlcNAc) as an internal standard. The samples were separated on a zwitterionic polymer-based high-performance liquid chromatography (HPLC) column before being fused into an electrospray ionization (ESI) single quadrupole
A
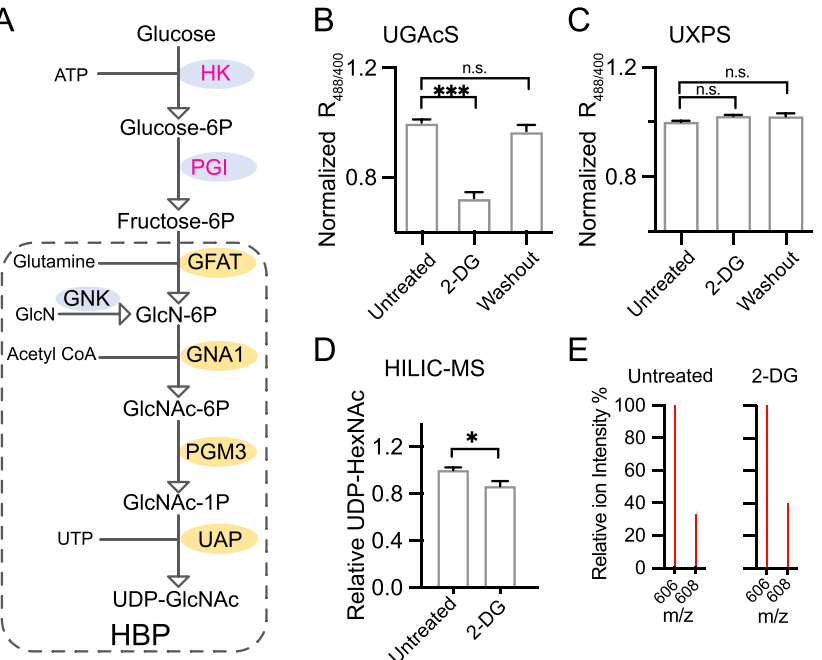

D

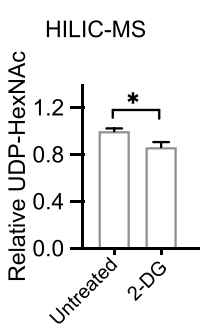

E



Figure 2. UDP-GlcNAc level changes in HEK 293T cells perturbed with 2-DG. (A) Schematic illustration of the hexosamine biosynthetic pathway (HBP) responsible for the production of UDP-GlcNAc. 2DG (2-deoxy-D-glucose) is a glycolysis inhibitor acting on hexokinase $(\mathrm{HK})$ and phosphoglucose isomerase (PGI), which are highlighted in magenta. Responses of UGAcS (B) or UXPS (C), given as normalized fluorescence excitation ratios $(488 \mathrm{~nm} / 400 \mathrm{~nm})$, to $3 \mathrm{~h}$ of incubation with $10 \mathrm{mM}$ 2-DG, or $3 \mathrm{~h}$ of $10 \mathrm{mM}$ 2-DG incubation followed by washout and an addition $3 \mathrm{~h}$ culture in normal medium. (D) HILICMS analysis of relative UDP-HexNAc concentrations in extracts of HEK $293 \mathrm{~T}$ cells untreated or treated with $10 \mathrm{mM}$ 2-DG. (E) Representative mass spectrograms of the samples in panel D. The $m / z$ $=606$ peak represents the major isotope peak for UDP-HexNAc. ${ }^{13} \mathrm{C}$ double-labeled UDP-GlcNAc $\left({ }^{13} \mathrm{C}_{2}\right.$-UDP-GlcNAc) was doped into the cell extracts as an internal standard, contributing primarily to the $m / z=608$ peak. Data in panels B-D are presented as mean \pm SEM ( $n=3$ wells of cells for each group). $P$ values were determined by one-way ANOVA with Dunnett's multiple comparisons test $(* * * P<$ 0.001 ; $* P<0.05$; and n.s., not significant, $P \geq 0.05$ ).

mass spectrometer. 606 and $608 \mathrm{~m} / z$ ions, which are the major isotope peaks for UDP-GlcNAc and ${ }^{13} \mathrm{C}_{2}$-UDP-GlcNAc, respectively, were monitored (Figure $\mathrm{S} 5$ ). The intensity ratio of the two peaks is thus an indicator for the relative UDPGlcNAc concentrations in the cell lysates. Since our chromatographic condition did not separate UDP-GlcNAc from UDPGalNAc, and the two nucleotide sugars have identical molecular formulas, the HILIC-MS method in fact measured UDP-GlcNAc and UDP-GalNAc (referred to as UDPHexNAc) collectively. Specific epimerases are responsible for the interconversion of UDP-GlcNAc and UDP-GalNAc in cells. The concentration ratio of UDP-GlcNAc to UDPGalNAc is usually $\sim 3: 1{ }^{32}$ Thus, the UDP-HexNAc measurements from HILIC-MS can be used to approximate UDPGlcNAc level changes. The HILIC-MS analysis confirmed that 2 -DG reduced the UDP-HexNAc level by $\sim 13.4 \%$ (Figure $2 \mathrm{D}, \mathrm{E})$, and the result corroborates the UGAcS-based fluorescence assay.

The glutamine fructose-6-phosphate aminotransferase (GFAT) is a feedback-regulated rate-limiting enzyme in the $\mathrm{HBP}^{33}$ Since glucosamine (GlcN) enters the HBP downstream of GFAT (Figure 2A), GlcN is a potent stimulator of $\mathrm{HBP}$ and can rapidly increase the intracellular concentration of UDP-GlcNAc. ${ }^{20}$ We used a confocal microscope equipped with 488 and $405 \mathrm{~nm}$ lasers to follow GlcN-stimulated UDPGlcNAc elevation in HEK 293 T cells. Upon GlcN stimulation, 
the fluorescence with $488 \mathrm{~nm}$ excitation increased along with the simultaneous decrease of the fluorescence with $405 \mathrm{~nm}$ excitation (Figure 3A,B), resulting in an overall $\sim 70 \%$
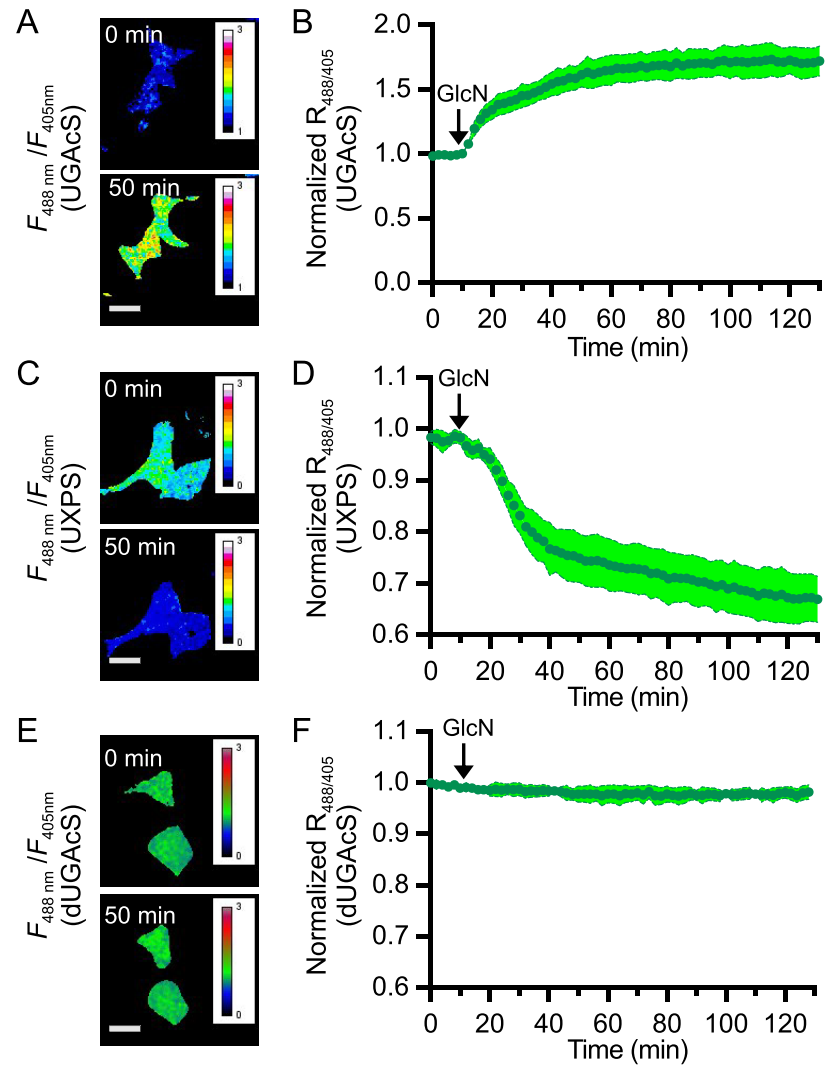

Figure 3. Glucosamine (GlcN)-induced UDP-GlcNAc increase in HEK 293T cells. Representative ratiometric images of HEK 293T cell expressing UGAcS (A), UXPS (C), or dUGAcS (E) with two excitation wavelengths $(488 \mathrm{~nm} / 405 \mathrm{~nm})$ before and after treatment with $5 \mathrm{mM}$ GlcN (scale bars, $40 \mu \mathrm{m}$ ). Quantitative traces of normalized fluorescence excitation ratios $(488 \mathrm{~nm} / 405 \mathrm{~nm})$ for UGAcS (B), UXPS (D), or dUGAcS (F) in HEK 293T cells. Data are presented as mean \pm SD $(n=10$ cells from 3 replicating wells for each group).

ratiometric change $\left(\Delta R / R_{0}\right)$. Most of the change was completed within the first 30 min poststimulation. In contrast, GlcN triggered an $\sim 33 \%$ ratiometric change $\left(\Delta R / R_{0}\right)$ of the UXPS fluorescence in the opposite direction (Figure 3C,D). The observed fluorescence change of UXPS is not surprising because the GlcN-dependent UDP-GlcNAc synthesis process may consume UTP quickly. Although the opposite responses of UGAcS and UXPS can exclude the possibility of the observed UGAcS response being caused by $\mathrm{pH}$ changes, we further introduced Arg164Ala and Glu269Ala double mutations into UXPS, resulting in a deactivated mutant (termed dUGAcS) unresponsive to UDP-GlcNAc, UTP, and UDP (Figure S6). We tested dUGAcS-expressing HEK 293T cells against GlcN stimulation and observed no response (Figure $3 \mathrm{E}, \mathrm{F})$. Collectively, these results confirmed that GlcN indeed increased the intracellular UDP-GlcNAc concentration and that UGAcS successfully detected this relatively rapid increase of UDP-GlcNAc in HEK 293T cells.

We next utilized the biosensors to examine UDP-GlcNAc concentration changes in response to the genetic disruption of two key enzymes in the HBP. GFAT is the first and rate- limiting enzyme, while UDP-N-acetylglucosamine pyrophosphorylase (UAP) is the last enzyme in the pathway and is responsible for the direct synthesis of UDP-GlcNAc (Figure 4A). We used short hairpin RNAs (shRNAs) to knock down

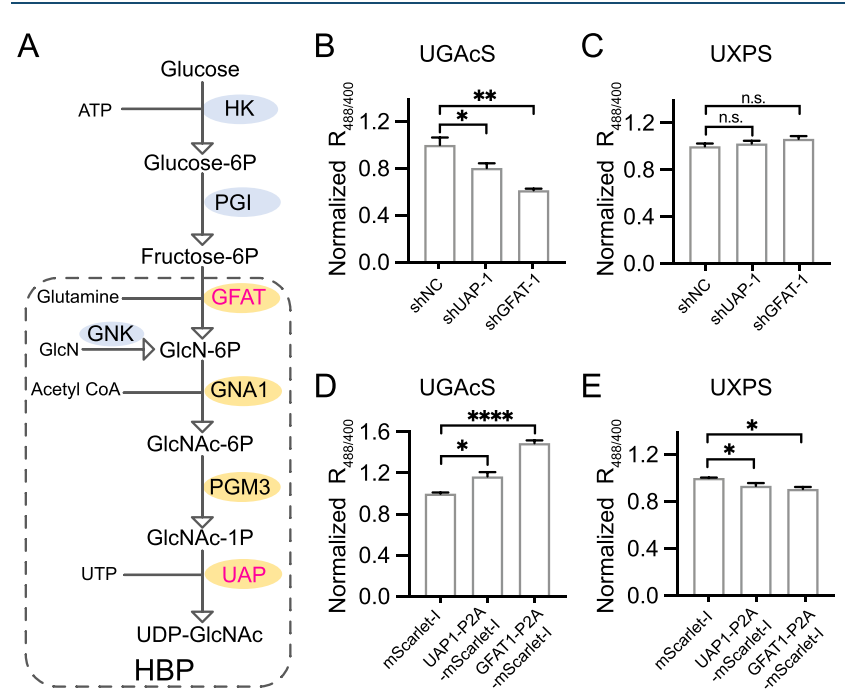

Figure 4. UDP-GlcNAc level changes in response to knockdown or overexpression of GFAT or UAP in HEK 293T cells. (A) Schematic illustration of the HBP UDP-GlcNAc synthesis pathway. Highlighted in magenta are the two enzymes selected for genetic manipulation in this study. Responses of UGAcS (B) or UXPS (C), presented as normalized fluorescence excitation ratios $(488 \mathrm{~nm} / 400 \mathrm{~nm})$, to shRNA knockdown of GFAT or UAP. A negative shRNA control, shNC, was used for comparison and normalization. Responses of UGAcS (D) or UXPS (E), given as normalized fluorescence excitation ratios $(488 \mathrm{~nm} / 400 \mathrm{~nm})$, to the overexpression of UAP1P2A-mScarlet-I or GFAT1-P2A-mScarlet-I. The overexpression of $\mathrm{mScarlet-I}$ alone was used for comparison and normalization. Data in panels B-E are presented as mean \pm SEM ( $n=3$ wells of cells for each group). $P$ values were determined by one-way ANOVA with Dunnett's multiple comparisons test $(* * * * P<0.0001$; $* * P<0.01$; $* P<0.05$; and n.s., not significant, $P \geq 0.05$ ).

GFAT or UAP. The effectiveness of the shRNAs was first confirmed using fluorescence assays with HEK 293T cells coexpressing corresponding shRNAs and the GFAT1 or UAP1 gene fused to a red fluorescent protein (RFP) mScarlet-I via a P2A self-cleaving peptide, as well as reverse transcriptionquantitative PCR (RT-qPCR) assays (Figure S7). The GFAT and UAP shRNA variants that induced the most significant decrease of $\mathrm{mScarlet}-\mathrm{I}$ fluorescence and mRNA abundance were selected for further experiments. A scramble nontargeting shRNA sequence ( $\mathrm{shNC}$ ) was included as a negative control. Next, we used the shRNA lentiviral vectors to infect HEK $293 \mathrm{~T}$ cells, to which the gene of UGAcS or UXPS was further introduced by transfection. As expected, GFAT or UAP knockdown decreased the UDP-GlcNAc level, as the fluorescence excitation ratios $\left(R_{488 / 400}\right)$ of UGAcS were reduced compared to the shNC control group (Figure $4 \mathrm{~B}$ ). Meanwhile, the fluorescence excitation ratios $\left(R_{488 / 400}\right)$ of UXPS in the experimental groups were slightly higher (statistically insignificant) than the shNC control group (Figure 4C). Furthermore, the same response trend was observed when GFAT was pharmacologically inhibited using DON (6-diazo-5-oxo-L-norleucine) (Figure S8).

We also examined the impact of overexpressing GFAT1 or UAP1 on the UDP-GlcNAc level. The sensor responses are 


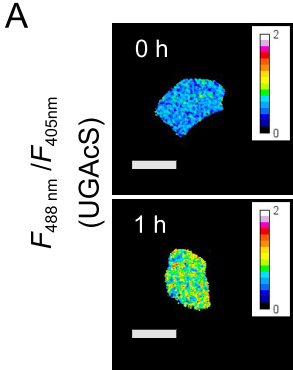

E

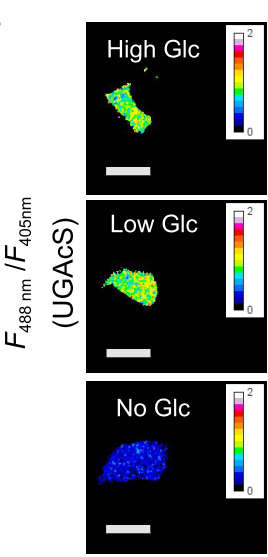

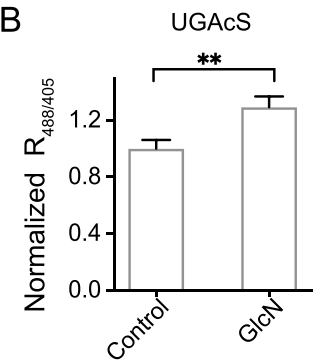

C

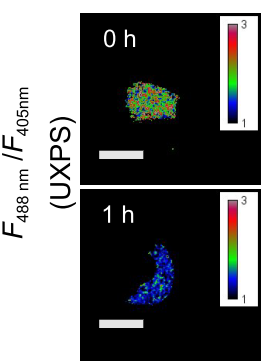

F
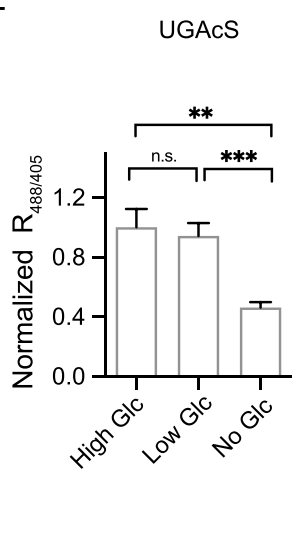

G



D

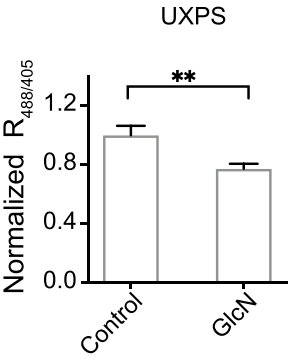

$\mathrm{H}$

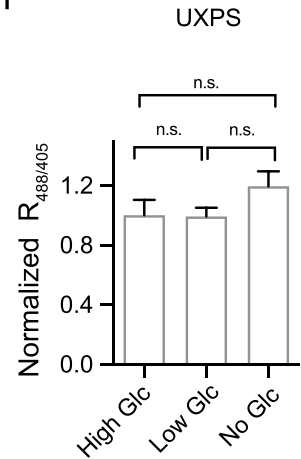

Figure 5. Imaging of UDP-GlcNAc levels in pancreatic MIN6 $\beta$-cells. Representative ratiometric images of a MIN6 cell expressing UGAcS (A) or UXPS (C) with two excitation wavelengths $(488 \mathrm{~nm} / 405 \mathrm{~nm}$ ) before and after a $1 \mathrm{~h}$ treatment with $5 \mathrm{mM}$ GlcN (scale bars, $40 \mu \mathrm{m})$. Responses of UGAcS (B) or UXPS (D), given as normalized fluorescence excitation ratios (488 nm/405 nm), to $1 \mathrm{~h}$ of incubation with $5 \mathrm{mM}$ GlcN. Representative ratiometric images of MIN6 cells expressing UGAcS (E) or UXPS (G) with two excitation wavelengths (488 nm/405 nm) cultured in high $(25 \mathrm{mM})$, low $(2 \mathrm{mM})$, or no glucose DMEM (scale bars, $40 \mu \mathrm{m}$ ). Responses of UGAcS (F) or UXPS (H), given as normalized fluorescence excitation ratios $(488 \mathrm{~nm} / 405 \mathrm{~nm})$, after replacing high glucose $(25 \mathrm{mM})$ medium with low $(2 \mathrm{mM})$ or no glucose medium for $20 \mathrm{~h}$. Data in panels $\mathrm{B}, \mathrm{D}, \mathrm{F}$, and $\mathrm{H}$ are presented as mean $\pm \operatorname{SEM}(n=12$ cells from 3 replicating wells for each group). $P$ values in panels B and D were determined by an unpaired two-tailed $t$-test $(* * P<0.01) . P$ values in panels $\mathrm{F}$ and $\mathrm{H}$ were determined by one-way ANOVA with Tukey's multiple comparisons test $(* * * P<0.001$; $* * P<0.01$; and n.s., not significant, $P \geq 0.05)$.

opposite to those in the knockdown experiments: GFAT1 or UAP1 overexpression increased the fluorescence excitation ratios $\left(R_{488 / 400}\right)$ of UGAcS and decreased the fluorescence excitation ratios $\left(R_{488 / 400}\right)$ of UXPS (Figure $\left.4 \mathrm{D}, \mathrm{E}\right)$. Moreover, in both the knockdown and overexpression experiments, manipulating the GFAT1 expression level induced more dramatic fluorescence responses than manipulating UAP1. This is again expected because GFAT plays the rate-limiting role in the HBP.

Imaging of UDP-GICNAc Levels in Pancreatic MIN6 $\beta$ Cells. Pancreatic $\beta$-cells are responsible for the synthesis and secretion of insulin, a key endocrine regulator of glucose levels in the blood and other tissues. ${ }^{34}$ The glucose metabolism of pancreatic $\beta$-cells is tightly coupled to insulin synthesis and secretion. ${ }^{34}$ Meanwhile, $O$-GlcNAc levels in $\beta$-cells have been linked to the regulation of insulin gene expression, proinsulinto-insulin processing, and glucose-stimulated insulin secretion. $^{35-37}$ In this context, we used our new biosensors to examine UDP-GlcNAc levels in MIN6 $\beta$-cells, a mouse insulinoma cell line, in response to various nutritional conditions. Expression of the biosensors in MIN6 cells resulted in bright green fluorescence. Upon $5 \mathrm{mM}$ GlcN stimulation, the fluorescence excitation ratios $\left(R_{488 / 400}\right)$ of UGAcSexpressing cells increased by $\sim 30 \%$ (Figure $5 \mathrm{AB}$ ) within $1 \mathrm{~h}$. In contrast, an $\sim 25 \%$ decrease was observed for UXPSexpressing cells (Figure 5CD), and no response was observed for dUGAcS-expressing cells (Figure S9A,B). The results suggest that GlcN, which bypasses the rate-limiting GFAT in the HBP, can stimulate the biosynthesis of UDP-GlcNAc in MIN6 cells in a manner similar to that in HEK 293T cells.

Next, MIN6 cells expressing these biosensors were subjected to glucose deprivation (replacing $25 \mathrm{mM}$ glucose medium with $2 \mathrm{mM}$ or no glucose medium) and imaged $20 \mathrm{~h}$ later. The fluorescence excitation ratio $\left(R_{488 / 400}\right)$ of UGAcS under the no glucose condition was $\sim 50 \%$ lower than that under the $25 \mathrm{mM}$ glucose condition, while we only observed a quite minimal decrease (statistically insignificant) of the fluorescence excitation ratio $\left(R_{488 / 400}\right)$ of UGAcS from the $25 \mathrm{mM}$ to the $2 \mathrm{mM}$ glucose condition (Figure 5E,F). The fluorescence changes of UXPS (Figure 5G,H) or dUGAcS (Figure S9C,D) were not significant under all conditions. We further examined the time course of UGAcS fluorescence changes in MIN6 cells from 25 to $0 \mathrm{mM}$ glucose and found that $10 \mathrm{~h}$ of glucose deprivation was adequate to complete all fluorescence changes (Figure S10). Taken together, the UDP-GlcNAc level in MIN6 cells was sensitive to severe hypoglycemia but relatively insensitive to glucose concentration changes from 25 to 2 $\mathrm{mM}$ within the examined time frame. These findings corroborate a recent study on the mouse heart tissue with 5.5 and $25 \mathrm{mM}$ glucose, which concluded that glucose availability alone does not regulate the HBP flux. ${ }^{3}$ In other studies, UDP-GlcNAc has been shown to directly inhibit GFAT via a negative feedback mechanism. ${ }^{33,38}$ The tight regulation of the $\mathrm{HBP}$, however, does not necessarily 
undermine the importance of UDP-GlcNAc in nutritional signaling. First, multiple types of metabolic molecules are funneled into the HBP, and a collection of these molecules may be needed to drastically shift the HBP flux. In addition, the GFAT expression level and activity are highly responsive to other signals, and the HBP is thus likely regulated to different extents in diverse tissue types and under various pathophysiological conditions. ${ }^{39-41}$

\section{CONCLUSION}

We have engineered a genetically encoded UDP-GlcNAc sensor (UGAcS) by inserting cpGFP into an inactivated UDPGlcNAc transferase. Because UGAcS is also responsive to UDP and UTP, we further engineered a control sensor, UXPS, which is only responsive to UDP and UTP but not UDPGlcNAc. We successfully applied the biosensors to monitoring UDP-GlcNAc level changes in HEK 293T cells in response to 2-DG-induced glycolysis inhibition, glucosamine stimulation, DON-induced GFAT inhibition, and the genetic manipulation of two key enzymes (GFAT and UAP) in the HBP. Finally, we used our biosensors to monitor UDP-GlcNAc levels in pancreatic MIN6 $\beta$-cells under various cell culture conditions. Our results suggest that glucose metabolism via the HBP is tightly regulated across a large glucose concentration range. Further research is clearly needed to further understand the regulatory mechanism of the HBP flux, and the fluorescent biosensors described here should facilitate these studies.

In addition to being used as research tools in studying the crucial roles of UDP-GlcNAc in disease and normal conditions, the biosensors may have translational applications. Modulation of the HBP has been considered as a promising method to treat diseases, such as cancer and diabetes. ${ }^{42,43}$ We envision the use of the biosensors to screen for chemical or genetic modulators of the HBP. In addition, murG is a key enzyme for peptidoglycan synthesis in bacteria, ${ }^{44}$ so these murG-based biosensors may be used to discover novel murG inhibitors as a new class of antibiotics.

Our effort to develop the first genetically encoded nucleotide sugar sensor will spur the development of future biosensors. UDP and UTP interfere with the response of UGAcS. Although UXPS can be used for cross-checking, a subset of treatment conditions may move the fluorescence ratios of both sensors toward the same direction, and it would become difficult to interpret the results. In addition, the interference makes the quantitative measurement of intracellular UDP-GlcNAc levels difficult. Directed evolution, machine learning, and computation-assisted design may be combined to further tune the specificity of UGAcS. Also, it may be possible to use alternative strategies to develop fluorescent UDP-GlcNAc sensors with different selectivity profiles. Furthermore, future research may lead to biosensors with altered affinities, in additional fluorescence colors, and for other important nucleotide sugars and carbohydrates.

Moreover, nucleotides, such as UTP and UDP, are important cell metabolites involved in diverse processes, such as nucleotide biosynthesis, transcription, purinergic signaling, and apoptotic cell clearance. ${ }^{45-47}$ Previous studies have developed several synthetic fluorescent indicators for UTP and UDP. ${ }^{48-50}$ Our genetically encoded UXPS will complement these efforts and be useful new tools for visualizing UTP and UDP dynamics in living systems.

\section{ASSOCIATED CONTENT}

\section{(1) Supporting Information}

The Supporting Information is available free of charge at https://pubs.acs.org/doi/10.1021/acscentsci.1c00745.

Additional experimental methods and figures including structures, $\mathrm{C}_{\alpha}$ dihedral differences, process flowchart, sequence alignment, HILIC-MS analysis, characterizations, mRNA abundance, responses, ratiometric images, and time-course fluorescence (PDF)

\section{Accession Codes}

The plasmids for bacterial and mammalian expression of UGAcS and UXPS and their sequence information have been deposited to Addgene (Plasmids 172135, 172136, 172137, and 172138).

\section{AUTHOR INFORMATION}

\section{Corresponding Author}

Hui-wang Ai - Department of Molecular Physiology and Biological Physics, and Center for Membrane and Cell Physiology, University of Virginia, Charlottesville, Virginia 22908, United States; orcid.org/0000-0002-5664-3270; Email: huiwang.ai@virginia.edu

Authors

Zefan Li - Department of Molecular Physiology and Biological Physics, and Center for Membrane and Cell Physiology, University of Virginia, Charlottesville, Virginia 22908, United States

Jing Zhang - Department of Molecular Physiology and Biological Physics, and Center for Membrane and Cell Physiology, University of Virginia, Charlottesville, Virginia 22908, United States

Complete contact information is available at:

https://pubs.acs.org/10.1021/acscentsci.1c00745

\section{Author Contributions}

J.Z. constructed and evaluated the plasmids for shRNA knockdown. Z.L. performed all other experiments, including sensor engineering and characterization, analyzed data, prepared figures, and wrote the manuscript. H.-w.A. supervised research, revised figures, and wrote the manuscript.

\section{Notes}

The authors declare no competing financial interest.

\section{ACKNOWLEDGMENTS}

Research reported in this publication was supported by the NIH Common Fund Glycoscience Program and the National Cancer Institute of the National Institutes of Health under Award U01CA230817. The content is solely the responsibility of the authors and does not necessarily represent the official views of the National Institutes of Health. pMD2.G (Addgene Plasmid 12259) and psPAX2 (Addgene Plasmid 12260) were gifts from Didier Trono (EPFL). pLKO.1 (Addgene Plasmid 10878) was a gift from David Root (Broad Institute). We also thank Yu Pang for the independent replication of some results in Figure 1, and Dr. Yiyu Zhang for assisting in culturing MIN6 cells.

\section{REFERENCES}

(1) Bond, M. R.; Hanover, J. A. A little sugar goes a long way: the cell biology of O-GlcNAc. J. Cell Biol. 2015, 208 (7), 869. 
(2) Yang, X.; Qian, K. Protein O-GlcNAcylation: emerging mechanisms and functions. Nat. Rev. Mol. Cell Biol. 2017, 18 (7), 452.

(3) Olson, A. K.; Bouchard, B.; Zhu, W. Z.; Chatham, J. C.; Des Rosiers, C. First characterization of glucose flux through the hexosamine biosynthesis pathway (HBP) in ex vivo mouse heart. $J$. Biol. Chem. 2020, 295 (7), 2018.

(4) Marshall, S.; Bacote, V.; Traxinger, R. R. Discovery of a metabolic pathway mediating glucose-induced desensitization of the glucose transport system. Role of hexosamine biosynthesis in the induction of insulin resistance. J. Biol. Chem. 1991, 266 (8), 4706.

(5) Ong, Q.; Han, W.; Yang, X. O-GlcNAc as an Integrator of Signaling Pathways. Front. Endocrinol. (Lausanne, Switz.) 2018, 9, 599.

(6) Akella, N. M.; Ciraku, L.; Reginato, M. J. Fueling the fire: emerging role of the hexosamine biosynthetic pathway in cancer. BMC Biol. 2019, 17 (1), 52.

(7) Flynn, R. A.; Pedram, K.; Malaker, S. A.; Batista, P. J.; Smith, B. A. H.; Johnson, A. G.; George, B. M.; Majzoub, K.; Villalta, P. W.; Carette, J. E.; et al. Small RNAs are modified with N-glycans and displayed on the surface of living cells. Cell 2021, 184 (12), 3109.

(8) Hanover, J. A. Epigenetics gets sweeter: O-GlcNAc joins the "histone code". Chem. Biol. 2010, 17 (12), 1272.

(9) Ramirez, D. H.; Aonbangkhen, C.; Wu, H. Y.; Naftaly, J. A.; Tang, S.; O’Meara, T. R.; Woo, C. M. Engineering a ProximityDirected O-GlcNAc Transferase for Selective Protein O-GlcNAcylation in Cells. ACS Chem. Biol. 2020, 15 (4), 1059.

(10) Hao, Y.; Fan, X.; Shi, Y.; Zhang, C.; Sun, D. E.; Qin, K.; Qin, W.; Zhou, W.; Chen, X. Next-generation unnatural monosaccharides reveal that ESRRB O-GlcNAcylation regulates pluripotency of mouse embryonic stem cells. Nat. Commun. 2019, 10 (1), 4065

(11) Qin, W.; Lv, P.; Fan, X.; Quan, B.; Zhu, Y.; Qin, K.; Chen, Y.; Wang, C.; Chen, $X$. Quantitative time-resolved chemoproteomics reveals that stable O-GlcNAc regulates box C/D snoRNP biogenesis. Proc. Natl. Acad. Sci. U. S. A. 2017, 114 (33), E6749.

(12) Pekkurnaz, G.; Trinidad, J. C.; Wang, X.; Kong, D.; Schwarz, T. L. Glucose regulates mitochondrial motility via Milton modification by O-GlcNAc transferase. Cell 2014, 158 (1), 54.

(13) Wani, W. Y.; Chatham, J. C.; Darley-Usmar, V.; McMahon, L. L.; Zhang, J. O-GlcNAcylation and neurodegeneration. Brain Res. Bull. 2017, 133, 80.

(14) Baumann, D.; Wong, A.; Akhaphong, B.; Jo, S.; Pritchard, S.; Mohan, R.; Chung, G.; Zhang, Y.; Alejandro, E. U. Role of nutrientdriven O-GlcNAc-post-translational modification in pancreatic exocrine and endocrine islet development. Development 2020, 147 (7), dev186643.

(15) Ma, J.; Hart, G. W. Protein O-GlcNAcylation in diabetes and diabetic complications. Expert Rev. Proteomics 2013, 10 (4), 365.

(16) Ranjan, A.; Kalraiya, R. D. $\alpha 2,6$ sialylation associated with increased $\beta 1,6$-branched $\mathrm{N}$-oligosaccharides influences cellular adhesion and invasion. J. Biosci. 2013, 38 (5), 867.

(17) Edvardson, S.; Ashikov, A.; Jalas, C.; Sturiale, L.; Shaag, A.; Fedick, A.; Treff, N. R.; Garozzo, D.; Gerardy-Schahn, R.; Elpeleg, O. Mutations in SLC35A3 cause autism spectrum disorder, epilepsy and arthrogryposis. J. Med. Genet. 2013, 50 (11), 733.

(18) Kreppel, L. K.; Hart, G. W. Regulation of a Cytosolic and Nuclear O-glcnac Transferase: Role of the Tetratricopeptide Repeats. J. Biol. Chem. 1999, 274 (45), 32015.

(19) Taylor, R. P.; Parker, G. J.; Hazel, M. W.; Soesanto, Y.; Fuller, W.; Yazzie, M. J.; McClain, D. A. Glucose deprivation stimulates OGlcNAc modification of proteins through up-regulation of O-linked $\mathrm{N}$-acetylglucosaminyltransferase. J. Biol. Chem. 2008, 283 (10), 6050.

(20) Taylor, R. P.; Geisler, T. S.; Chambers, J. H.; McClain, D. A. Up-regulation of O-GlcNAc transferase with glucose deprivation in HepG2 cells is mediated by decreased hexosamine pathway flux. J. Biol. Chem. 2009, 284 (6), 3425.

(21) Slawson, C.; Copeland, R. J.; Hart, G. W. O-GlcNAc signaling: a metabolic link between diabetes and cancer? Trends Biochem. Sci. 2010, 35 (10), 547.

(22) Liu, K.; Paterson, A. J.; Chin, E.; Kudlow, J. E. Glucose stimulates protein modification by O-linked GlcNAc in pancreatic $\beta$ cells: Linkage of O-linked GlcNAc to $\beta$ cell death. Proc. Natl. Acad. Sci. U. S. A. 2000, 97 (6), 2820.

(23) Vasconcelos-dos-Santos, A.; de Queiroz, R. M.; da Costa Rodrigues, B.; Todeschini, A. R.; Dias, W. B. Hyperglycemia and aberrant O-GlcNAcylation: contributions to tumor progression. $J$. Bioenerg. Biomembr. 2018, 50 (3), 175.

(24) Ito, J.; Herter, T.; Baidoo, E. E.; Lao, J.; Vega-Sánchez, M. E.; Michelle Smith-Moritz, A.; Adams, P. D.; Keasling, J. D.; Usadel, B.; Petzold, C. J.; et al. Analysis of plant nucleotide sugars by hydrophilic interaction liquid chromatography and tandem mass spectrometry. Anal. Biochem. 2014, 448, 14.

(25) Nakajima, K.; Kitazume, S.; Angata, T.; Fujinawa, R.; Ohtsubo, K.; Miyoshi, E.; Taniguchi, N. Simultaneous determination of nucleotide sugars with ion-pair reversed-phase HPLC. Glycobiology 2010, 20 (7), 865.

(26) Crouvoisier, M.; Auger, G.; Blanot, D.; Mengin-Lecreulx, D. Role of the amino acid invariants in the active site of MurG as evaluated by site-directed mutagenesis. Biochimie 2007, 89 (12), 1498.

(27) Hu, Y.; Chen, L.; Ha, S.; Gross, B.; Falcone, B.; Walker, D.; Mokhtarzadeh, M.; Walker, S. Crystal structure of the MurG:UDPGlcNAc complex reveals common structural principles of a superfamily of glycosyltransferases. Proc. Natl. Acad. Sci. U. S. A. 2003, 100 (3), 845 .

(28) Mengin-Lecreulx, D.; Texier, L.; Rousseau, M.; van Heijenoort, J. The murG gene of Escherichia coli codes for the UDP-Nacetylglucosamine: $\mathrm{N}$-acetylmuramyl-(pentapeptide) pyrophosphorylundecaprenol $\mathrm{N}$-acetylglucosamine transferase involved in the membrane steps of peptidoglycan synthesis. J. Bacteriol. 1991, 173 (15), 4625.

(29) Chen, L.; Men, H.; Ha, S.; Ye, X. Y.; Brunner, L.; Hu, Y.; Walker, S. Intrinsic lipid preferences and kinetic mechanism of Escherichia coli MurG. Biochemistry 2002, 41 (21), 6824.

(30) Pajak, B.; Siwiak, E.; Soltyka, M.; Priebe, A.; Zieliński, R.; Fokt, I.; Ziemniak, M.; Jaśkiewicz, A.; Borowski, R.; Domoradzki, T.; et al. 2-Deoxy-d-Glucose and Its Analogs: From Diagnostic to Therapeutic Agents. Int. J. Mol. Sci. 2020, 21 (1), 234.

(31) Alpert, A. J. Hydrophilic-interaction chromatography for the separation of peptides, nucleic acids and other polar compounds. $J$. Chromatogr. A 1990, 499, 177.

(32) Robinson, K. A.; Weinstein, M. L.; Lindenmayer, G. E.; Buse, M. G. Effects of diabetes and hyperglycemia on the hexosamine synthesis pathway in rat muscle and liver. Diabetes 1995, 44 (12), 1438

(33) Ruegenberg, S.; Horn, M.; Pichlo, C.; Allmeroth, K.; Baumann, U.; Denzel, M. S. Loss of GFAT-1 feedback regulation activates the hexosamine pathway that modulates protein homeostasis. Nat. Commun. 2020, 11 (1), 687.

(34) Wilcox, G. Insulin and insulin resistance. Clin. Biochem. Rev. 2005, 26 (2), 19-39.

(35) Durning, S. P.; Flanagan-Steet, H.; Prasad, N.; Wells, L. OLinked $\beta$-N-acetylglucosamine (O-GlcNAc) Acts as a Glucose Sensor to Epigenetically Regulate the Insulin Gene in Pancreatic Beta Cells. J. Biol. Chem. 2016, 291 (5), 2107.

(36) Akimoto, Y.; Hart, G. W.; Wells, L.; Vosseller, K.; Yamamoto, K.; Munetomo, E.; Ohara-Imaizumi, M.; Nishiwaki, C.; Nagamatsu, S.; Hirano, $\mathrm{H}_{\text {.; }}$ et al. Elevation of the post-translational modification of proteins by $\mathrm{O}$-linked $\mathrm{N}$-acetylglucosamine leads to deterioration of the glucose-stimulated insulin secretion in the pancreas of diabetic Goto-Kakizaki rats. Glycobiology 2007, 17 (2), 127.

(37) Jo, S.; Lockridge, A.; Alejandro, E. U. eIF4G1 and carboxypeptidase $\mathrm{E}$ axis dysregulation in O-GlcNAc transferasedeficient pancreatic $\beta$-cells contributes to hyperproinsulinemia in mice. J. Biol. Chem. 2019, 294 (35), 13040.

(38) Teo, C. F.; Wollaston-Hayden, E. E.; Wells, L. Hexosamine flux, the O-GlcNAc modification, and the development of insulin resistance in adipocytes. Mol. Cell. Endocrinol. 2010, 318 (1-2), 44.

(39) Gélinas, R.; Mailleux, F.; Dontaine, J.; Bultot, L.; Demeulder, B.; Ginion, A.; Daskalopoulos, E. P.; Esfahani, H.; Dubois-Deruy, E.; 
Lauzier, B.; et al. AMPK activation counteracts cardiac hypertrophy by reducing O-GlcNAcylation. Nat. Commun. 2018, 9 (1), 374.

(40) Li, L.; Shao, M.; Peng, P.; Yang, C.; Song, S.; Duan, F.; Jia, D.; Zhang, M.; Zhao, J.; Zhao, R.; et al. High expression of GFAT1 predicts unfavorable prognosis in patients with hepatocellular carcinoma. Oncotarget 2017, 8 (12), 19205.

(41) Ruegenberg, S.; Mayr, F.; Atanassov, I.; Baumann, U.; Denzel, M. S. Protein kinase A controls the hexosamine pathway by tuning the feedback inhibition of GFAT-1. Nat. Commun. 2021, 12 (1), 2176.

(42) Walter, L. A.; Lin, Y. H.; Halbrook, C. J.; Chuh, K. N.; He, L.; Pedowitz, N. J.; Batt, A. R.; Brennan, C. K.; Stiles, B. L.; Lyssiotis, C. A.; et al. Inhibiting the Hexosamine Biosynthetic Pathway Lowers OGlcNAcylation Levels and Sensitizes Cancer to Environmental Stress. Biochemistry 2020, 59 (34), 3169.

(43) Buse, M. G. Hexosamines, insulin resistance, and the complications of diabetes: current status. Am. J. Physiol. Endocrinol. Metab. 2006, 290 (1), E1.

(44) Hu, Y.; Helm, J. S.; Chen, L.; Ginsberg, C.; Gross, B.; Kraybill, B.; Tiyanont, K.; Fang, X.; Wu, T.; Walker, S. Identification of selective inhibitors for the glycosyltransferase MurG via highthroughput screening. Chem. Biol. 2004, 11 (5), 703.

(45) Anderson, C. M.; Parkinson, F. E. Potential signalling roles for UTP and UDP: sources, regulation and release of uracil nucleotides. Trends Pharmacol. Sci. 1997, 18 (10), 387.

(46) Huang, Z.; Xie, N.; Illes, P.; Di Virgilio, F.; Ulrich, H.; Semyanov, A.; Verkhratsky, A.; Sperlagh, B.; Yu, S. G.; Huang, C.; et al. From purines to purinergic signalling: molecular functions and human diseases. Signal Transduct. Target Ther. 2021, 6 (1), 162.

(47) Elliott, M. R.; Ravichandran, K. S. The Dynamics of Apoptotic Cell Clearance. Dev. Cell 2016, 38 (2), 147.

(48) Chen, X.; Jou, M. J.; Yoon, J. An "Off-On" type UTP/UDP selective fluorescent probe and its application to monitor glycosylation process. Org. Lett. 2009, 11 (10), 2181.

(49) Kambam, S.; Ren, X.; Zheng, C.; Wang, F.; Wang, Y.; Chen, H.; Yin, J.; Xie, J.; Chen, X. The synthesis of UDP-selective fluorescent probe and its imaging application in living cells. Bioorg. Med. Chem. Lett. 2015, 25 (2), 262.

(50) Lv, X. W.; Wang, G. K.; Zou, X. J.; Xie, X. G.; Zhou, Y. Study of a 1,8-naphtylimide derivative as uridine diphosphate selective probe: Synthesis, optical properties and in vivo imaging application. Dyes Pigm. 2017, 142, 552. 